\title{
EFFECTS OF INGESTING MILK FERMENTED BY LACTOCOCCUS LACTIS H61 ON SKIN PROPERTIES AND HEALTH BIOMARKERS IN MIDDLE-AGED WOMEN: A RANDOMIZED, DOUBLE-BLIND STUDY
}

\author{
H. Kimoto-Nira, N. Moriya, K. Sasaki, C. Suzuki
}

\begin{abstract}
Objective: Previously, we showed that ingestion of fermented milk made by only Lactococcus lactis strain H61 (H61fermented milk) improves various skin properties in young Japanese women (age, around 20 y). Because the condition of human skin varies with age, we investigated the effects of H61-fermented milk on skin properties of middle-aged women. Design and setting: A randomized, double-blind trial in a clinical research setting. Participants: Healthy, middle-aged, female volunteers ( $\mathrm{n}=$ 23; age: 36-62 y). Intervention: H61-fermented milk (10 $\left.{ }^{10} \mathrm{CFU}\right)$ or conventional yogurt (10 ${ }^{10}$ CFU of both Lactobacillus delbrueckii subsp. bulgaricus and Streptococcus thermophilus; a reference food) was given daily for 4 weeks. Measurements: Before and at the end of the 4-week treatment, we measured skin hydration (inner forearms and cheek) and sebum content (cheek only). In addition, salivary samples were collected for analysis of immune, stress, and inflammation biomarkers. Self-questionnaires (regarding skin and general health conditions) were done at week 4. Results: By mechanical analysis, the interventions did not alter the skin properties of either group. At week 4, self-questionnaire skin scores for elasticity and texture were significantly higher; scores for darkness tended to be improved in the H61-fermented milk group than the conventional yogurt group. In the general health condition section, diarrhea was significantly more prevalent in the H61-fermented milk than in the conventional yogurt group. The intervention did not alter immunoglobulin A, cortisol, $\alpha$-amylase, and C-reactive protein levels in saliva. Conclusions: According to self-questionnaires, H61-fermented milk provides beneficial effects on some skin properties of middle-aged women. This intervention would be useful for increasing the quality of life in an aging population after characterizing the beneficial effect by mechanical analysis in future study.
\end{abstract}

Key words: Fermented milk, Lactococcus lactis, skin health.

\section{Introduction}

As the elderly population increases, the prevalence of aging-related, physiologic deterioration will increase; thus, functional foods that provide health benefits to control aging and prolong health will likely become more desirable. In this context, the probiotic properties of lactic acid bacteria have garnered particular interest. According to the Food and Agriculture Organization-World Health Organization, probiotics are «live microorganisms which, when administered in adequate amounts, confer a health benefit to the host». Specifically, several reports have addressed the effect of lactic acid bacteria (including fermented milk made by probiotic lactic acid bacteria)

NARO Institute of Livestock and Grassland Science, Ikenodai 2, Tsukuba, Ibaraki 305-0901, Japan

Corresponding Author: H. Kimoto-Nira, NARO Institute of Livestock and Grassland Science, Ikenodai 2, Tsukuba, Ibaraki 305-0901, Japan, tel: +81298388688, fax: +81298388606, e-mail: anne@affrc.go.jp by using aged animals. For example, a Lactobacillus casei strain Shirota activates immune responses in aged mice and ameliorates influenza viral infections (1), and Bifidobacterium- and Lactobacillus-fermented milk is beneficial in the treatment of inflammatory bowel disease, possibly via stabilization of mucosal immunity in senescence-accelerated mice (2). Furthermore, a certain lactic acid bacterium decreases the incidence of infection in elderly people (3).

Moreover, some compounds may improve aged skin in healthy people. This is of particular interest to women, whose quality of life may be affected by their skin health. According to our previous study in senescence-accelerated mice, oral administration of heatkilled Lactococcus lactis subsp. cremoris strain H61 was associated with suppressed external skin deterioration, such as skin ulcers and hair loss (4). In our trials using heat-killed strain H61 on middle-aged (approximately 50s-60s), healthy women, reduction of skin hydration 
(caused by seasonal change) was suppressed $(5,6)$. Thus, strain H61 may be useful for an aging society. The strain H61 has been widely used over the past 50 years in Japan to produce fermented dairy products; however, the dairy industry is interested in its effects on skin properties.

Kumagai et al. (7) reported that the physiological development of the skin reaches a stage of completion during 20s, and becomes comparatively stable, and thereafter (from the middle of 30s) gradually descend. Aged skin has several typical characteristics, including fine wrinkles, sallowness, and loss of elasticity (8). The skin of elderly people is generally thought to be dry ( 9 , 10). However, it was reported that adult women (age: 30-48 y) have higher skin hydration (in the cheek and inner forearm) than their children (age: 10-14 y) (11), or hydration of normal skin does not differ between young and elderly women (12). Although there are some contradictory reports, it is assumed that skin status is altered with age.

In our previous study, women (approximately 20 y) consumed fermented milk made with only L. lactis strain H61 (H61-fermented milk) for 4 weeks; they reported significantly improved skin status by self-questionnaires (13) and had increased sebum content in their cheeks by mechanical analysis (14). In the present study, to investigate the effects of H61-fermented milk on the skin properties of older women, we determined their skin status before and after intake of H61-fermented milk or conventional yogurt (as a reference) by using mechanical and self-questionnaire analyses. In addition, we measured immune (immunoglobulin A), stress $(\alpha$-amylase and cortisol), and inflammation (C-reactive protein) biomarkers in the saliva of our participants to elucidate factors possibly affecting skin properties.

\section{Materials and methods}

\section{Participants and study design}

This study was performed between February and March 2013 as a randomized (two groups), double-blind application test. Twenty-four women employed at our Institute, more than $35 \mathrm{y}$, were recruited by handbill. Exclusion criteria were pregnancy, breastfeeding, and routine use of medical products to treat various metabolic, cardiovascular, or hepatic diseases. One participant dropped out because she did not meet the inclusion criteria. Twenty-three middle-aged (age: 36-62 y) healthy women were enrolled. The mean ages were $49.7 \mathrm{y}$ and $48.6 \mathrm{y}$ in the H61-fermented milk group ( $\mathrm{n}=$ 12 ) and conventional yogurt group $(n=11)$, respectively; the age distribution was comparable between these groups.

The participants were randomly assigned to two groups according to the skin properties observed at week 0 and age. The study lasted 4 weeks; at weeks 0 and 4, we assessed skin parameters and collected saliva samples for analysis of biomarkers. This study was conducted according to the guidelines in the Declaration of Helsinki, and all procedures involving humans were approved by the ethics committees of the NARO Institute of Livestock and Grassland Science. All participants received detailed information about purposes, methods, expected results, and ethical considerations (including possible adverse effects relevant to the study) and submitted a written declaration of consent to participate in the study. This study was performed at Nishinasuno in Tochigi prefecture. Participants enrolled the study were instructed to stable cosmetic application before and during the study. They were also instructed to have stable life patterns of outdoor activity and food intake.

\section{Test foods}

H61-fermented milk and conventional yogurt were produced by Japan Agricultural Cooperatives Ibaraki Mizuho (Hitachiohta, Ibaraki, Japan) every 2 weeks and distributed to the participants. Both products contained glucose, fructose, and oligosaccharide; levels of all nutrients were similar except for the lactic acid bacteria used. H61-fermented milk was made by using only $L$. lactis subsp. cremoris H61 (MAFF 400007; The Genebank of National Institute of Agrobiological Sciences, Tsukuba, Japan) at a level of $10^{8} \mathrm{CFU} / \mathrm{mL}$. Conventional yogurt was made by using both Lactobacillus delbrueckii subsp. bulgaricus and Streptococcus thermophilus at a level of $10^{8} \mathrm{CFU} / \mathrm{mL}$ each as a starter culture (Chr. Hansen, Hoersholm, Denmark). Both products were packed in identical containers. Participants consumed $150 \mathrm{~mL}$ (1010 CFU level) of either H61-fermented milk or conventional yogurt daily; this is within the range of most clinical studies $\left(4 \times 10^{8}\right.$ to $\left.1 \times 10^{11} \mathrm{CFU}\right)(15,16)$. They were also instructed to record in a diary any symptoms experienced and/or medications taken during the 4-week intervention; this information was provided for the evaluation of safety.

The statistician generated a computerized randomization sequence. Participants and investigators were unaware of the treatment assignment. In addition, the evaluator of the results (i.e. statistician) was also not aware which treatment any particular participants received. The distribution of the test food was performed by a person without any practical involvement in the study and without being aware which is H61-fermented milk or conventional yogurt.

\section{Mechanical analyses of skin properties}

In study participants, we assessed the hydration of the skin of the right and left inner forearms and the hydration and sebum content of the lateral angles of the right and left cheeks. Prior to the measurements, each participant 
washed her cheeks once with identical soap in tap water and sat quietly for $20 \mathrm{~min}$ to equilibrate to the conditions in the testing room (approximately $20-24^{\circ} \mathrm{C}$ and $27-39 \%$ relative humidity). Two symmetrical regions of the cheeks were evaluated. Skin hydration was measured by using a corneometer (CM 825, Courage and Khazaka Electronics, Cologne, Germany) $(17,18)$. The sebum content was measured by using a Sebumeter SM810 (Courage and Khazaka Electronics) (18). Skin hydration was measured three times at each location and time point. Sebum content was measured once per time point.

\section{Self-questionnaire}

The self-evaluation questionnaire comprised two sections: (I) perceptions of skin-specific attributes and (II) indictors of general health (6). Specifically, section I addressed the following skin properties: moisture, smoothness, oiliness, elasticity, luster, texture, number and size of pimples, darkness, spots, redness, wrinkles (forehead, around eyes), sagging (around eyes, around mouth), ability to retain cosmetics, and apparent hair follicles. Section II included swelling, oversensitivity to cold, paleness of skin, dizziness, pain in waist, stiff neck, headache, hot flash, sweatiness, dryness of throat, constipation, tension of belly, diarrhea, tiredness, appetite, ease of falling asleep, physical strength, psychological strength, irritation, and luster of hair. Participants evaluated these conditions by responding to questionnaire items on a 5-point scale (1: the characteristic was markedly better than that of week $0 ; 3$ : no change in the characteristic; 5: the characteristic was markedly worse than that of week 0 ) for each item.

\section{Analysis of biomarkers in saliva}

The general status of the participants was evaluated by saliva analyses at the beginning and end of the 4 -week intervention period. Participants were instructed to refrain from eating $30 \mathrm{~min}$ before saliva sampling. At weeks 0 and 4, sampling was performed by using an oral swab (Salimetrics, State College, PA, USA); participants were sampled at the almost same time of day for both time points. Participants kept the swabs under their tongues for $5 \mathrm{~min}$ before putting each in a tube. Swabs were frozen at $-20^{\circ} \mathrm{C}$ and sent for analyses at a certified clinical laboratory (Funakoshi, Tokyo, Japan). Secretory immunoglobulin A (s-IgA), cortisol, and C-reactive protein (CRP) in saliva were analyzed by enzymelinked immunosorbent assay by using a commercial kit (Salimetrics). Alpha-amylase in saliva was measured by using the Dry Chemistry System (Nypro Corp., Osaka, Japan) (19), according to the manufacturer's protocol.

\section{Statistical analysis}

Data were expressed as mean or mean with standard deviation or standard error. For all skin properties evaluated by using mechanical analysis (at weeks 0 and 4), we calculated and analyzed descriptive statistics by using a general linear model procedure (SAS version

Table 1

Effects of conventional yogurt and H61-fermented milk intake on various skin parameters of middle-aged women

\begin{tabular}{|c|c|c|c|c|c|c|}
\hline & \multirow{2}{*}{\multicolumn{2}{|c|}{$\begin{array}{l}\text { Inner forearm } \\
\text { Hydration (A.U.) }\end{array}$}} & \multicolumn{4}{|c|}{ Cheek } \\
\hline & & & \multicolumn{2}{|c|}{ Hydration (A.U.) } & \multicolumn{2}{|c|}{ Sebum $(\mu \mathrm{g} / \mathrm{cm} 2)$} \\
\hline & $\mathrm{L}$ & $\mathbf{R}$ & L & $\mathbf{R}$ & L & $\mathbf{R}$ \\
\hline \multicolumn{7}{|l|}{ Group } \\
\hline Yogurt & $36.9(2.2)$ & $40.4(2.0)$ & $53.9(2.2)$ & $52.3(1.8)$ & $5.40(3.1)$ & $2.49(0.9)$ \\
\hline H61 & $38.5(2.1)$ & $39.5(1.9)$ & $52.1(2.1)$ & $53.0(1.7)$ & $5.75(3.0)$ & $3.21(0.9)$ \\
\hline$P$ value & 0.604 & 0.762 & 0.552 & 0.796 & 0.936 & 0.583 \\
\hline \multicolumn{7}{|l|}{ Time point } \\
\hline Before intake & $36.7(2.1)$ & $39.6(1.9)$ & $54.9(2.1)$ & $53.6(1.7)$ & $6.93(3.0)$ & $2.32(0.9)$ \\
\hline After intake & $38.6(2.1)$ & 40.3 (1.9) & $51.0(2.1)$ & $51.7(1.7)$ & $4.23(3.0)$ & $3.38(0.9)$ \\
\hline P value & 0.531 & 0.812 & 0.206 & 0.463 & 0.536 & 0.416 \\
\hline \multicolumn{7}{|c|}{ Group $\times$ time point } \\
\hline Yogurt Before & $35.8(3.1)$ & $41.2(2.8)$ & $57.0(3.0)$ & $53.5(2.5)$ & $8.85(4.4)$ & $2.30(1.3)$ \\
\hline Yogurt After & $38.0(3.1)$ & $39.5(2.8)$ & $50.7(3.0)$ & $51.2(2.5)$ & $1.95(4.4)$ & $2.68(1.3)$ \\
\hline H61 Before & $37.6(2.9)$ & $38.0(2.7)$ & $52.8(2.9)$ & $53.7(2.4)$ & $5.00(4.2)$ & $2.33(1.3)$ \\
\hline H61 After & $39.3(2.9)$ & $41.0(2.7)$ & $51.3(2.9)$ & $52.3(2.4)$ & $6.50(4.2)$ & $4.08(1.3)$ \\
\hline$P$ value & 0.932 & 0.401 & 0.422 & 0.855 & 0.340 & 0.600 \\
\hline
\end{tabular}

A.U., arbitrary units. Values are expressed as least-squares means. Figures in parentheses are standard errors. L, left side. R, right side. 
Table 2

Analysis of self-questionnaires on skin and general health conditions after the intervention

\begin{tabular}{|c|c|c|c|c|c|}
\hline \multirow[b]{2}{*}{ Item } & \multicolumn{2}{|c|}{ Conventional yogurt } & \multicolumn{2}{|c|}{ H61-fermented milk } & \multirow[b]{2}{*}{ P value } \\
\hline & Mean & SD & Mean & SD & \\
\hline \multicolumn{6}{|l|}{ Skin condition } \\
\hline Moisture & 2.82 & 0.41 & 2.50 & 0.80 & 0.213 \\
\hline Smoothness & 2.82 & 0.41 & 2.42 & 0.67 & 0.105 \\
\hline Oiliness & 3.00 & 0.45 & 2.92 & 0.29 & 0.599 \\
\hline Elasticity & 3.00 & 0.00 & 2.67 & 0.49 & 0.039 \\
\hline Luster & 2.91 & 0.30 & 2.67 & 0.49 & 0.168 \\
\hline Texture & 3.00 & 0.00 & 2.67 & 0.49 & 0.039 \\
\hline Pimples (number, size) & 3.00 & 0.45 & 2.92 & 0.29 & 0.599 \\
\hline Darkness & 3.00 & 0.00 & 2.75 & 0.45 & 0.082 \\
\hline Spots (number, size) & 3.00 & 0.00 & 3.00 & 0.00 & 1.000 \\
\hline Redness & 2.91 & 0.30 & 2.75 & 0.45 & 0.325 \\
\hline Wrinkles (forehead) & 3.00 & 0.00 & 2.83 & 0.39 & 0.166 \\
\hline Wrinkles (around eyes) & 2.73 & 0.65 & 2.92 & 0.29 & 0.462 \\
\hline Sagging (around eyes) & 2.82 & 0.41 & 2.75 & 0.45 & 0.699 \\
\hline Sagging (around mouth) & 2.91 & 0.30 & 2.83 & 0.39 & 0.598 \\
\hline Ability to retain cosmetics & 3.00 & 0.00 & 3.00 & 0.00 & 1.000 \\
\hline Apparent hair follicles & 2.82 & 0.41 & 2.83 & 0.39 & 0.925 \\
\hline Total skin condition & 2.73 & 0.65 & 2.50 & 0.52 & 0.402 \\
\hline \multicolumn{6}{|l|}{ Body condition } \\
\hline Swelling & 2.91 & 0.70 & 2.83 & 0.39 & 0.402 \\
\hline Oversensitivity to cold & 2.91 & 0.30 & 2.83 & 0.39 & 0.598 \\
\hline Paleness of skin & 2.91 & 0.30 & 2.92 & 0.29 & 0.950 \\
\hline Dizziness & 2.82 & 0.41 & 3.00 & 0.00 & 0.131 \\
\hline Pain in waist & 3.09 & 0.30 & 3.00 & 0.00 & 0.296 \\
\hline Stiff neck & 3.00 & 0.00 & 3.00 & 0.43 & 1.000 \\
\hline Headache & 2.91 & 0.30 & 2.83 & 0.58 & 1.000 \\
\hline Hot flash & 2.91 & 0.30 & 3.00 & 0.00 & 0.296 \\
\hline Sweatiness & 3.00 & 0.00 & 3.00 & 0.00 & 1.000 \\
\hline Dryness of throat & 3.00 & 0.00 & 2.83 & 0.58 & 0.338 \\
\hline Constipation & 3.00 & 0.63 & 2.58 & 1.17 & 0.210 \\
\hline Tension of belly & 3.36 & 0.67 & 3.08 & 0.52 & 0.226 \\
\hline Diarrhea & 3.00 & 0.00 & 3.42 & 0.67 & 0.040 \\
\hline Tiredness & 3.00 & 0.00 & 2.92 & 0.29 & 0.338 \\
\hline Appetite & 3.00 & 0.45 & 3.00 & 0.00 & 1.000 \\
\hline Ease of falling asleep & 3.00 & 0.00 & 2.92 & 0.29 & 0.338 \\
\hline Sound sleep & 3.00 & 0.45 & 2.92 & 0.29 & 0.599 \\
\hline Physical strength & 2.82 & 0.41 & 2.92 & 0.29 & 0.493 \\
\hline Psychologic strength & 3.00 & 0.00 & 3.00 & 0.00 & 1.00 \\
\hline Irritation & 2.82 & 0.41 & 2.83 & 0.39 & 0.925 \\
\hline Luster of hair & 2.91 & 0.30 & 2.92 & 0.29 & 0.950 \\
\hline Total body condition & 2.73 & 0.47 & 2.75 & 0.75 & 0.761 \\
\hline
\end{tabular}

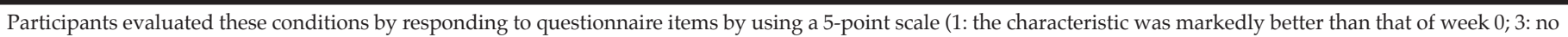
change in the characteristic; 5: the characteristic was markedly worse than that of week 0) for each item. Data are expressed as mean values with SD. 
9.1, SAS Institute, Cary, NC, USA). Least-square means were compared by applying the Tukey-Kramer test. Self-questionnaire scores for skin and general health conditions between the two groups were analyzed by using the Kruskal-Wallis test. $P<0.05$ was considered statistically significant, and $P<0.1$ indicated a trend.

\section{Results}

Participants demonstrated good compliance with the study requirements and according to their diaries, the two treatment groups did not differ with respect to test drink intake. All subjects who participated in this study were evaluated in the present analysis. Importantly, severe adverse effects or symptoms were not observed.

For the mechanical analyses of skin properties, we investigated the skin hydration and sebum content of middle-aged women who consumed conventional yogurt or H61-fermented milk; neither group nor time point affected any skin parameters (Table 1). Interaction between group and time point had no effect on the hydration of the inner forearm or cheek or sebum content in either group.

For the self-questionnaires, scores are shown for skin and general health conditions after intervention (Table 2 ). In the skin condition section, scores for elasticity and texture in the H61-fermented milk group were significantly higher than in the conventional yogurt group $(P<0.05)$. The score for darkness in the H61fermented milk group tended to be lower (i.e. skin was self-reported to be lighter in color) than in the conventional yogurt group $(P<0.1)$. In the general health condition section, according to self-reported scores, diarrhea was significantly more prevalent in the H61fermented milk than in the conventional yogurt group $(P$ $<0.05)$.

Finally, we analyzed immune, stress, and inflammation parameters in the saliva (Table 3). For our immune parameter, the groups tended to have different s-IgA levels $(P<0.1)$ (Table 3$)$; however, a significant difference was not observed between the groups and time points. Furthermore, the intervention did not significantly affect our stress parameters, $\alpha$-amylase and cortisol, or our inflammation parameter, CRP.

\section{Discussion}

The consumption of various foods is thought to affect skin conditions $(20,21)$. For example, increased intake of vitamin $\mathrm{C}$ and linoleic acid and decreased consumption of fats and carbohydrates are associated with improved appearance in the skin of aging women (22). However, there are few studies on the effect of fermented milk (including yogurt) on the condition of the skin of healthy humans. Previously, we conducted a double-blinded trial evaluating the effect of H61-fermented milk and conventional yogurt on the skin properties of young (approximately 20 y) women; by mechanical analysis, the sebum content in the cheek rose significantly after intervention in the H61-fermented milk group but not the conventional yogurt group (14). In addition, in other study, after 4 weeks of consuming yogurt made by

Table 3

Effects of conventional yogurt and H61-fermented milk intake on stress, immune, and inflammation biomarker levels in middle-aged women

\begin{tabular}{lcccc}
\hline & $\begin{array}{c}\alpha \text {-amylase } \\
(\mathbf{K U} / \mathbf{L})\end{array}$ & $\begin{array}{c}\text { Cortisol } \\
(\mu \mathrm{g} / \mathrm{dL})\end{array}$ & $\begin{array}{c}\text { Immunogloblin A } \\
(\mu \mathrm{g} / \mathrm{mL})\end{array}$ & $\begin{array}{c}\text { C-reactive protein } \\
(\mathbf{p g} / \mathbf{m L})\end{array}$ \\
\hline Group & & & & $241(29)$ \\
Yogurt & $31.8(4.4)$ & $0.21(0.04)$ & $166(27)$ & $3184(526)$ \\
H61 & $29.3(4.2)$ & $0.29(0.03)$ & 0.072 & 0.167 \\
P value & 0.676 & 0.115 & & \\
Time point & & & $198(28)$ & $2344(539)$ \\
Before intake & $35.0(4.3)$ & $0.25(0.03)$ & $209(28)$ & $2929(539)$ \\
After intake & $26.1(4.3)$ & $0.25(0.03)$ & 0.804 & 0.453 \\
P value & 0.159 & 0.908 & & $2064(780)$ \\
Group $\times$ time point & & & $247(41)$ & $3743(780)$ \\
Yogurt Before & $37.0(6.2)$ & $0.22(0.05)$ & $236(41)$ & $2625(744)$ \\
Yogurt After & $26.7(6.2)$ & $0.20(0.05)$ & $150(39)$ & $3743(744)$ \\
H61 Before & $33.0(5.9)$ & $0.28(0.05)$ & $181(39)$ & 0.493 \\
H61 After & $25.5(5.9)$ & $0.30(0.05)$ & 0.597 & \\
P value & 0.818 & 0.662 & & \\
\hline
\end{tabular}


using Lactobacillus delbrueckii subsp. bulgaricus 2038 and Streptococcus thermophilus 1131, Japanese women (20 -39 y) skin elasticity (by mechanical and self-questionnaire analyses) increased and the degree of cheek dryness decreased (by self-questionnaire) (23). However, those studies were performed in younger people; therefore, the effect of fermented milk on the skin properties of older people may be of particular interest for societies with increasing aging populations. In our present study, we compared the effects of H61-fermented milk with conventional yogurt, as a reference food, on the skin health of older people. A reference food is needed because of seasonal effects (e.g., environmental changes such as temperature and humidity), which may alter skin conditions. In middle-aged women (36-62 y), intake of H61-fermented milk may show beneficial effects on skin properties (skin elasticity, texture, and darkness) by self-questionnaire analysis. Therefore, similar to the effects on young women, H61-fermented milk would likely be beneficial for the skin of older people. The beneficial effects of milk on skin status in human have been reported. For example, whole milk intake was associated with alleviation for atopic dermatitis (24). In our previous study (4), administration of strain H61 as living cells suspended in milk, or a fermented milk made with the strain to mice was found to reduce skin trouble with aging (i.e. incidence of hair loss and skin ulcers) compared with mice taken milk, and there was no significant difference in skin status between living cells and fermented milk. Considering that those results in mice can be extrapolated to those in human, and heat-killed cells provides beneficial effect on skin status in women $(5,6)$, milk and milk metabolites produced by strain $\mathrm{H} 61$ are unlikely to be associated with improvement of skin status. In the present study, it is suggested that difference of skin evaluation between H61-fermented milk and conventional yogurt is attributed to kinds of lactic acid bacteria included.

To investigate the mechanisms for our observed effects regarding the intake of H61-fermented milk on skin properties, we measured several biomarkers in saliva. Saliva collection is relatively non-invasive; importantly, the characteristics of saliva can be a marker of a person's health and well-being. Specifically, s-IgA in saliva has been widely used as an indicator of mucosal immunity (25) and decreases with age $(26,27)$. Moreover, immune responses are closely associated with skin properties. For example, exposure to UVB (280-320 nm) can impair specific and nonspecific immune responses (28); UVB-induced immunomodulation contributes to photocarcinogenesis (29). In the present study, intake of H61-fermented milk and conventional yogurt for 4 weeks did not affect s-IgA, indicating that they could not change mucosal immune responses.

Skin health in humans is also affected by psychologic stress (30). Biomarkers of stress include salivary $\alpha$-amylase and cortisol $(19,31)$, which are secreted under psychologic and physical stress. For example, compared with those who sleep well, children who sleep poorly display higher diurnal $\alpha$-amylase and cortisol levels throughout the entire day (32). In the present study, considering that salivary $\alpha$-amylase and cortisol were not altered by the intervention, intake of H61-fermented milk and conventional yogurt may not be effective in the amelioration of stress in middle-aged women. These results correspond with our self-questionnaire analysis for stress status (irritation and psychologic strength, Table 2). There are mixed results in the field regarding s-IgA and stress: salivary s-IgA is elevated by acute psychologic stressors (31), and s-IgA levels are increased by short-term relaxation (33). Furthermore, there are also mixed results regarding the intake of H61-fermented milk and stress: in our study, H61-fermented milk likely did not affect s-IgA as a biomarker of short-term relaxation or stress, while others report that irritation in young women (by self-questionnaire) is improved by intake of H61-fermented milk (13). Therefore, in future studies, the relationship between stress status and intake of H61fermented milk should be analyzed with other stress biomarkers.

Lastly, we investigated a biomarker of inflammation, CRP; serum CRP is often used in the risk assessment of some diseases, such as coronary events (34) and periodontosis (35). In a study of 61 healthy adults, there was a moderate to strong association between CRP in serum and saliva (36). In contrast, salivary and serum CRP levels were not correlated in a group of 55 healthy young adults (37). In the present study, salivary CRP was not altered by the intervention; no detectable inflammation likely occurred in the participants regardless of the relationship between serum and salivary CRP.

Skin and intestinal health are associated (23). For example, according to a self-questionnaire analysis, H61fermented milk decreases the incidence of constipation and diarrhea in young women (13). In our present study, the incidence of constipation decreased in the H61-fermented milk group than conventional yogurt group; however, significance was not achieved (Table 2). Furthermore, diarrhea worsened in the H61-fermented milk group compared with the conventional yogurt group. It is likely that the effects on constipation and diarrhea are a similar phenomenon. Therefore, altered of participants' intestinal condition due to their intake of H61-fermented milk may contribute to the observed improvement in several skin properties.

It was reported that women with the appearance of wrinkles, with senile dryness, and with skin atrophy were related with postmenopausal (22). In terms of skin hydration and sebum content analyzed in our study, Nagata et al. (18) reported that there is no correlation between postmenopausal and skin hydration, sebum content. However, information of premenopausal and postmenopausal for the subjects was not included in the 
present study. It is a subject for further study to consider postmenopausal status and skin parameters.

In conclusion, H61-fermented milk altered skin properties (e.g., improvement of elasticity and texture) in middle-aged, healthy women more than conventional yogurt did by self-surveyed test. This probiotic strain would be useful for increasing the quality of life in an aging population. As far oral intake of strain H61, we will intend to confirm skin items with the obtained improvement by self-evaluation, via mechanical analysis with appropriate measurable instrument in a larger participant population.

Acknowledgments: We thank all of the volunteers who participated in this study.

Conflict of Interest: The authors declare no conflict of interests associated with this manuscript.

Ethics Statement: This study was conducted according to the guidelines in the Declaration of Helsinki, and all procedures were approved by the ethics committees of the NARO Institute of Livestock and Grassland Science.

\section{References}

1. Hori T, Kiyoshima J, Shida K, Yasui H. Augmentation of cellular immunity and reduction of influenza virus titer in aged mice fed Lactobacillus casei strain Shirota. Clin Diagn Lab Immunol 2002;9: 105-108.

2. Matsumoto S, Watanabe N, Imaoka A, Okabe Y. Preventive effects of Bifidobacterium-and Lactobacillus-fermented milk on the development of inflammatory bowel disease in senescence-accelerated mouse P1/Yit strain mice. Digestion 2001;64: 92-99.

3. Fukushima Y, Miyaguchi S, Yamano T, Kaburagi T, Iino H, Ushida K, Sato K Improvement of nutritional status and incidence of infection in hospitalised enterally fed elderly by feeding of fermented milk containing probiotic Lactobacillus johnsonii La1 (NCC533). Br J Nutr 2007;98: 696-977.

4. Kimoto-Nira H, Suzuki C, Kobayashi M et al. Anti-aging effect of a lactococcal strain: analysis using senescence-accelerated mice. Br J Nutr 2007;98: 1178-1186.

5. Kimoto-Nira H, Aoki R, Sasaki K, Suzuki C, Mizumachi K. Effect of Oral Intake of a Lactococcus lactis Strain on Skin Properties of Women-a Pilot Study-.Nihon Chikusan Gakkaihou 2012a;83: 307-313.

6. Kimoto-Nira H, Aoki R, Sasaki $\mathrm{K}$ et al. Oral intake of heat-killed cells of Lactococcus lactis strain H61 promotes skin health in women. J Nutr Sci 2012b;1: e19 (7 pages).

7. Kumagai H, Watanabe H, Kozu T, Noguchi H, Takahashi M. Age-related changes in the skin of Japanese women. J Soc Cosmet Chem Japan 1989;23:921.

8. Makrantonaki E, Zouboulis CC. Characteristics and pathomechanisms of endogenously aged skin. Dermatology 2007;214: 352-360.

9. Potts RO, Buras EM, Chrisman DA. Changes with age in the moisture content of human skin. J Invest Dermatol 1984;82: 97-100.

10. Rogiers V, Derde MP, Verleye G et al. Standardized conditions needed for skin surface hydration measurements. Cosmet Toilet 1990;105: 73-82.

11. Akutsu N, Ooguri M, Onodera T et al. Functional characteristics of the skin surface of children approaching puberty: age and seasonal influences. Acta Derm Venereol 2009;89: 21-27.

12. Frodin T, Helander P, Molin L et al. Hydration of human stratum corneum studied in vivo by optothermal infrared spectrometry, electrical capacitance measurement, and evaporimetry. Acta Derm Venereol 1988;68: 461-467.

13. Suzuki Y, Kimoto-Nira H, Nagakura Y et al. Effect of yoghurt fermented by Lactococcus lactis ssp. cremoris H61 on defecation, skin and health conditions of female college student. New Diet Therapy 2013;29: 23-30.

14. Kimoto-Nira H, Nagakura $\mathrm{Y}$, Kodama $\mathrm{C}$ et al. Effects of ingesting fermented milk by using Lactococcus lactis H61 on skin health in young women: A randomized double-blind study. J Dairy Sci 2014;97: 5898-5903.

15. Larsen, C. N., S. Nielsen, P. Kastel et al. Dose-response study of probiotic bacteria Bifidobacterium animalis subsp. lactis BB-12 and Lactobacillus paracasei subsp. paracasei CRL-341 in healthy young adults. Eu J Clin Nutr 2006;60: 1284-1293.

16. Savard, P., B. Lamarche, M. E. Paradis et al. Impact of Bifidobacterium animalis subsp. lactis BB-12 and Lactobacillus acidophilus LA-5-containing yoghurt, on fecal bacterial counts of healthy adults. Int J Food Microbiol 2011;149: 50-57.

17. De Spirt S, Stahl W, Tronnier H et al. Intervention with flaxseed and borage oil supplements modulates skin condition in women. Br J Nutr 2009;101: 440-445.

18. Nagata C, Nakamura K, Wada K et al. Association of dietary fat, vegetables, and antioxidant micronutrients with skin ageing in Japanese women. $\mathrm{Br} J$ Nutr 2010;103: 1493-1498.

19. Maruyama Y, Kawano A, Okamoto $S$ et al. Differences in salivary alphaamylase and cortisol responsiveness following exposure to electrical stimulation versus the trier social stress tests. PLos ONE 2012;7: e39375.

20. Heinrich U, Neukam K, Tronnier $\mathrm{H}$ et al. Long-term ingestion of high flavonol cocoa provides photoprotection against UV-induced erythema and improves skin condition in women. J Nutr 2006;136: 1565-1569.

21. Stahl W, Heinrich U, Aust $\mathrm{O}$ et al. Lycopene-rich products and dietary photoprotection. Photonem Photobiol Sci 2006;5: 238-242.

22. Cosgrove $\mathrm{MC}$, Franco $\mathrm{OH}$, Stewart PG et al. Dietary nutrients intakes and skin-aging appearance among middle-aged American women. Am J Clin Nutr 2007;86: 1225-1231.

23. Isawa K, Noma T, Yamamoto $M$ et al. Verifying the ability of yogurt prepared with LB81 lactic acid bacteria to improve skin function. J Int Microbiol 2008;22: 1-5.

24. Keller S, Le HY, Rodiger C Hipler UC, Kertscher R, Malarski A et al. Supplementation of a dairy drink enriched with milk phospholipids in patients with atopic dermatitis- a double-blind, placebo-controlled, randomized, cross-over study. Clin Nutr 2014;S0261-5614 (14) 00039-9.

25. Alberts R, Antonie JM, Bourdet-Sicard R, Calder PC, Gleeson M, Lesourd B, Samartin S, Sanderson IR, Van Loo J, Vas Dias FW, Watzl B. Markers to measure immunomodulation in human nutrition intervention studies. Br J Nutr 2005;94: 452-481.

26. Smith DJ, Joshipura K, Kent R, Taubman MA. Effect of age on immunoglobulin content and volume of human labial gland saliva. J Dent Res 1992;71:1891-1894.

27. Miletic ID, Schiffman SS, Miletic VD, Sattely-Miller EA. Salivary IgA secretion rate in young and elderly persons. Physiol Behav 60:243-248.

28. Hurks M, Garssen I, van Loveren H et al. Ge1996;neral aspects of UV-irradiation on the immune system. In: Photobiology in Medicine, 1994;p. 161 [G. Jori, editor]. New York: Plenum Press.

29. Kripke ML. Immunologic mechanisms in UV-radiation carcinogenesis. Adv Cancer Res 1989;34: 69-106.

30. Heller MM, Lee ES, Koo JY. Stress as an influencing factor in psoriasis. Skin Therapy Lett 2011;16: 1-4.

31. Takatsuji K, Sugimoto Y, Ishizaki S, Ozaki Y, Matsuyama E, Yamaguchi Y. The effects of examination stress on salivary cortisol, immunoglobulin $\mathrm{A}$, and chromogranin A in nursing students. Biomed Res 2008;27: 221-224.

32. Raikkonen $\mathrm{K}$ et al. Poor sleep and altered hypothalamic-pituitaryadrenocortical and sympatho-adrenal-medullary system activity in children. J Clin Endocrinol Metab 2010;95: 2254-2261.

33. Taniguchi T, Hirokawa K, Tsuchiya M, Kawakami N. The immediate effects of 10-minute relaxation training on salivary immunoglobulin $\mathrm{A}(\operatorname{sIg} \mathrm{A})$ and mood state for Japanese female medical co-workers. Acta Med Okayama 2007;61:139-145.

34. Wilson AM, Ryan MC, Boyle AJ. The novel role of C-reactive protein in cardiovascular disease: risk marker or pathogen. Int. J. Cardiol. 2006;106: 291.

35. Kanaparthy R, Kanaparthy A, Mahendra M. C-reactive protein as a marker of periodontal disease. Gen Dent. 2012;60: e1-5.

36. Ouellet-Morin I, Danese A, Williams B, Arseneault L. Validation of a highsensitive assay for C-reactive protein in human saliva. Brain Behav Immun 25: 640-646.

37. Dillon MC, Opris DC, Kopanczyk R et al. Detection of homocystein and C-reactive protein in the saliva of healthy adults: comparison with blood levels. Biomark Insight. 2010;5: 57. 